\title{
SUMMING LOGS OF THE VELOCITY IN NRQCD AND TOP THRESHOLD PHYSICS *
}

\author{
ANDRÉ H. HOANG \\ Max-Planck-Institut für Physik \\ (Werner-Heisenberg-Institut), \\ Föhringer Ring 6, \\ 80805 München, Germany \\ E-mail: ahoang@mppmu.mpg.de
}

\begin{abstract}
To achieve reliable predictions of the top-antitop threshold cross section at a future $e^{+} e^{-}$Linear Collider logarithms of the top velocity need to be resummed. I review the issues that make this problem complicated and show how the task can be achieved by renormalization in an effective theory using the so called velocity renormalization group. The most recent NNLL order results are discussed.
\end{abstract}

\section{Introduction}

The so-called "threshold scan" of the total cross section line-shape of top pair production constitutes a major part of the top quark physics program at a future $e^{+} e^{-}$Linear Collider. ${ }^{1}$ From the location of the rise of the cross section a precise measurement of the top quark mass with experimental uncertainties below $100 \mathrm{MeV}$ will be gained, while from the shape and the normalization of the cross section one can extract the top quark Yukawa coupling $y_{t}$ (for a light Higgs), the top width or the strong coupling. ${ }^{2}$

In the threshold region, $\sqrt{s} \simeq 2 m_{t} \pm 10 \mathrm{GeV}$, the top quarks move with nonrelativistic velocity in the c.m. frame. Due to the large top width, $\Gamma_{t} \approx 1.5 \mathrm{GeV}$, toponium resonances cannot form and the cross section is a smooth function of the c.m. energy. This also means that non-perturbative effects can be neglected for predictions of the total cross section. It is convenient to define the top velocity by $m_{t} v^{2} \equiv \sqrt{s}-2 m_{t}$. Because in the loop expansion one encounters terms proportional to $\left(\alpha_{s} / v\right)^{n}$ from the instantaneous exchange of $n$ time-like gluons, one has to count $v \sim \alpha_{s}$

*Invited talk at Continuous Advances in QCD 2004, Minneapolis, USA May 13-16, 2004. Preprint Number: MPP-2004-153 
and to carry out an expansion in $\alpha_{s}$ as well as in $v$. Schematically the perturbative expansion of the R-ratio for the total top-antitop threshold cross section, $R=\sigma_{t \bar{t}} / \sigma_{\mu^{+} \mu^{-}}$, has the form

$$
R=v \sum_{k=0}^{\infty}\left(\frac{\alpha_{s}}{v}\right)^{k} \times\left\{1(\mathrm{LO}) ; \alpha_{s}, v(\mathrm{NLO}) ; \alpha_{s}^{2}, \alpha_{s} v, v^{2}(\mathrm{NNLO})\right\} .
$$

The expansion scheme in Eq. (1) is called fixed-order expansion, although it actually involves summations of the terms proportional to $\left(\alpha_{s} / v\right)^{n}$ to all orders. The scheme can be implemented systematically using the factorization properties of Non-relativistic QCD (NRQCD) established by Lepage etal. ${ }^{3}$ The NNLO QCD corrections to the total cross section were calculated already some time ago. $4,5,6,7,8,9,10$ One of the important results was that in a threshold top quark mass scheme (the most common schemes in use are the kinetic, PS and 1S mass schemes ${ }^{11}$ ) the theoretical uncertainties for a top mass measurement are comparable to the experimental uncertainties. However, it was also found that the NNLO corrections to the normalization of the cross section were as sizeable as the NLO corrections leading to a theoretical normalization uncertainty of at least $20 \% .{ }^{13}$ It was shown ${ }^{12,13}$ that this normalization uncertainty does not affect significantly the top mass measurement since the latter is mainly sensitive to the energy were the cross section rises. However, this normalization uncertainty jeopardizes competitive measurements of the top width, strong top coupling, and the top Yukawa coupling. Figure 1a shows the vector-current-induced R-ratio $\sigma\left(e^{+} e^{-} \rightarrow \gamma^{*} \rightarrow t \bar{t}\right) / \sigma\left(e^{+} e^{-} \rightarrow \mu^{+} \mu^{-}\right)$at LO, NLO and NNLO in the fixed-order expansion for typical choices of parameters and renormalization scales. ${ }^{a}$ It is the discrepancy between the seemingly well behaved NLO curves and the NNLO results which is the worrying issue.

One way to understand the large NNLO corrections is to recall that the $t \bar{t}$ pair at threshold is non-relativistic and that its dynamics is governed by vastly different energy scales, the top mass ( $m_{t} \sim 175 \mathrm{GeV}$, "hard"), the top three-momentum $(\mathbf{p} \simeq m v \simeq 25 \mathrm{GeV}$, "soft" $)$ and the top kinetic or potential energy $\left(E \simeq m v^{2} \simeq 3-4 \mathrm{GeV}\right.$, "ultrasoft"). The top width $\Gamma_{t} \approx 1.5 \mathrm{GeV}$, which protects against non-perturbative effects (and which is the main reason rendering the top threshold a precision observable) is of order $E$, but can be neglected for most of the following conceptual considerations. This hierarchy of scales is the basis of NRQCD factorization, ${ }^{3}$ which

${ }^{a}$ The contributions originating from Z-exchange are about an order of magnitude smaller and can be neglected as far as discussions on QCD uncertainties are concerned. 
separates short-distance physics at the scale $m$ from long-distance physics at the non-relativistic scales $\mathbf{p}$ and $E$. In its original formulation NRQCD has one quantum field for each of the non-relativistic quarks and antiquarks and one quantum field for the low-energy gluons. NRQCD matrix elements and Wilson coefficients therefore involve three types of logarithmic terms,

$$
\ln \left(\frac{\mu^{2}}{m_{t}^{2}}\right), \quad \ln \left(\frac{\mu^{2}}{\mathbf{p}^{2}}\right), \quad \ln \left(\frac{\mu^{2}}{E^{2}}\right) .
$$

These logarithms cannot be rendered small (or summed up) for any single choice of the renormalization scale $\mu$. However, this can be severe: for example, $\alpha_{s}\left(m_{t}\right) \ln \left(m_{t}^{2} / E^{2}\right) \simeq 0.8$ for $\mu=m_{t}$ in the case of top quark pair production close to threshold. Obviously, a more sophisticated effective field theory (EFT) framework has to be devised to resolve this issue and to allow to resum all logarithmic terms.

In this talk I discuss some of the conceptual aspects of an effective theory that is capable of systematically summing all logarithms of the velocity that can appear in the description of the non-relativistic quark-antiquark dynamics. The tool needed is called the velocity renormalization group $(\mathrm{VRG})^{14}$ and it is the basis of a renormalization group improved perturbative expansion. To be specific, I will concentrate mostly on an EFT known as vNRQCD. ${ }^{14,15,16,17}$. At the end I will come back to the top threshold cross section in order to review the present status.

\section{The Proper Effective Theory}

To begin with, it is useful to summarize the properties one desires from an effective theory of non-relativistic quark-antiquark pairs:

(1) IR (on-shell) fluctuations, including IR divergences, in the full theory are reproduced by EFT fields. This avoids large logarithms in matching conditions.

(2) There is a well-defined and systematic power counting scheme (in $v$ ). It can be uniquely identified which operators to account for what to compute at a given order. Moreover, all EFT loop integrations are governed by only one single scale.

(3) There is a consistent renormalization prescription to treat UV divergences and to formulate anomalous dimension which, eventually, will allow to sum all "large" logarithmic terms.

(4) All symmetries (spin, gauge, etc.) are implemented. This gives the most predictive power. 
(5) The Lagrangian is formulated in the regulator-independent way.

The original NRQCD ${ }^{3}$ does in fact not offer properties 2 and 3 . This is because there is one single gluon field describing soft and ultrasoft gluon effects, while the power counting for soft and ultrasoft gluon effects differs. For summing logarithms of the type shown above this is a severe obstacle. For example, soft gluons are participating in the binding of the quark pair while ultrasoft gluons are responsible for retardation effects such as the Lamb shift, and their interactions have to be multipole expanded. The situation is, however, even more complicated because, although $v \ll 1$ ensures that there is a strong hierarchy $m \gg m v \gg m v^{2}$, the soft and ultrasoft scales turn out to be correlated by the energy-momentum relation of the massive top quarks, $E \sim \mathbf{p}^{2} / m_{t}$.

One approach in the literature to resolve these issues has been to ignore the correlation of soft and ultrasoft scales at the beginning and to account for the scale hierarchy $m \gg m v \gg m v^{2}$ in the traditional Wilsonian (stepwise) way. One starts with the NRQCD by Lepage etal. having soft gluons and then "integrates out" soft effects at the scale $m v$. This results in nonlocal heavy quark interactions, the potentials, and interactions of the heavy quarks with the remaining ultrasoft gluons. The theory below $m v$ is called pNRQCD ${ }^{18}$ (p for "potential") and naturally avoids any double counting of gluon effects. In the NRQCD-pNRQCD approach there is no unique power counting. A v counting taking into account the energy-momentum relation of the massive quarks exists in pNRQCD, while in the intermediate NRQCD theory a $1 / m$ counting is applied and the heavy quarks are static. A particular property of pNRQCD is that the correlation of the soft and ultrasoft scales has eventually to be implemented back into the theory when UV-divergences in massive quark loops occur. Renormalization group running caused by such divergences then also couples the ultrasoft renormalization scale together with the soft matching scale back to the hard scale $m_{t} \cdot{ }^{19}$

In an alternative approach one accounts for the correlation of the soft and the ultrasoft scales from the very beginning which then forbids using a step-wise approach for the hierarchy of the momentum and the energy scale. Rather, there is only one EFT below the scale $m_{t}$ which then contains simultaneously soft and ultrasoft gluons. This approach is called vNRQCD ${ }^{14,15,16,17}$ (v for "velocity") and allows for a consistent v power counting that obeys the correlation $E \sim \mathbf{p}^{2} / m_{t}$ at all scales below $m_{t}$. There is an ultrasoft renormalization scale, $\mu_{U}$, for loops dominated by the 
ultrasoft energy scale, and a soft renormalization scale, $\mu_{S}$, for loops dominated by the soft momentum scale which also includes the massive quark loops. Both renormalization scales are correlated, $\mu_{U}=\mu_{S}^{2} / m=m \nu^{2}$. The running of coefficients and operators in the EFT is then expressed in terms of the dimension-zero scaling parameter $\nu$, where $\nu=1$ corresponds to the hard matching scale and $\nu=v$ is the low-energy scale where matrix elements are computed. The resulting renormalization group scaling, describing the correlated running coming from soft and ultrasoft effects is called the VRG ${ }^{14}$. As long as one does not have to account for UV divergences coming from massive quark loops there is a rather straightforward correspondence between the operator structure in vNRQCD and the one in the NRQCD-pNRQCD approach.

The effective vNRQCD Lagrangian (defined in the c.m. frame) is build from heavy potential quarks and antiquarks $\left(\psi_{\mathbf{p}}, \chi_{\mathbf{p}}\right)$, soft gluons, ghosts, and massless quarks $\left(A_{q}^{\mu}, c_{q}, \varphi_{q}\right)$ and ultrasoft gluons, ghosts, and massless quarks $\left(A^{\mu}, c, \varphi_{u s}\right)$. Double counting for the gluon effects is avoided since ultrasoft gluons reproduce only the physical gluon poles where $k^{0} \sim$ $\mathbf{k} \sim m v^{2}$, while soft gluons only have poles with $k^{0} \sim \mathbf{k} \sim m v$. All soft loops are made infrared-finite and at the same time all ultrasoft divergences in ultrasoft loops are made correspond to the hard scale $m$ by the pull-up

mechanism. ${ }^{20}$ It is essential that both soft and ultrasoft gluons are included at all scales below $m$ because the heavy quark equation of motion correlates the soft and ultrasoft scales. The dependences on soft energies and momenta of the heavy quark and soft gluon fields appear as labels on the fields, while only the lowest-energy ultrasoft fluctuations are associated by an explicit coordinate dependence. Formally this is achieved by a phase redefinition for the potential and soft fields ${ }^{14}, \phi(x) \rightarrow \sum_{k} e^{-i k \cdot x} \phi_{k}(x)$, where $k$ denotes momenta $\sim m v$ and $\partial^{\mu} \phi_{k}(x) \sim m v^{2} \phi_{k}(x)$.

\section{The Effective Theory Action}

The effective vNRQCD Lagrangian for a non-relativistic $t \bar{t}$ in its c.m. frame in a angular momentum S-wave and color singlet state has terms ${ }^{14,15,16}$

$$
\begin{aligned}
\mathcal{L} & =\sum_{\mathbf{p}}\left\{\psi_{\mathbf{p}}^{\dagger}\left[i D^{0}+i \frac{\Gamma_{t}}{2}+\delta m_{t}-\frac{(\mathbf{p}-i \mathbf{D})^{2}}{2 m_{t}}+\ldots\right] \psi_{\mathbf{p}}+(\psi \rightarrow \chi)\right\} \\
& -\frac{1}{4} G^{\mu \nu} G_{\mu \nu}-\mu_{S}^{2 \epsilon} g_{s}^{2} \sum_{\mathbf{p}, \mathbf{p}^{\prime}, q, q^{\prime}, \sigma}\left\{\frac{1}{2} \psi_{\mathbf{p}^{\prime}}^{\dagger}\left[A_{q^{\prime}}^{\mu}, A_{q}^{\nu}\right] U_{\mu \nu}^{(\sigma)} \psi_{\mathbf{p}}+(\psi \rightarrow \chi)+\ldots\right\}
\end{aligned}
$$




$$
\begin{aligned}
& +\sum_{p}\left|p^{\mu} A_{p}^{\nu}-p^{\nu} A_{p}^{\mu}\right|^{2}+\ldots-\sum_{\mathbf{p}, \mathbf{p}^{\prime}} \mu_{S}^{2 \epsilon} V\left(\mathbf{p}, \mathbf{p}^{\prime}\right) \psi_{\mathbf{p}^{\prime}}^{\dagger} \psi_{\mathbf{p}} \chi_{-\mathbf{p}^{\prime}}^{\dagger} \chi_{-\mathbf{p}} \\
& +\sum_{\mathbf{p}, \mathbf{p}^{\prime}} \frac{2 i \mu_{S}^{2 \epsilon} \mathcal{V}_{c}}{\left(\mathbf{p}^{\prime}-\mathbf{p}\right)^{4}} f^{A B C}\left(\mathbf{p}-\mathbf{p}^{\prime}\right) \cdot\left(\mu_{U}^{\epsilon} g_{u} \mathbf{A}^{C}\right)\left[\psi_{\mathbf{p}^{\prime}}^{\dagger} T^{A} \psi_{\mathbf{p}} \chi_{-\mathbf{p}^{\prime}}^{\dagger} \bar{T}^{B} \chi_{-\mathbf{p}}\right]+\ldots
\end{aligned}
$$

where color and spin indices have been suppressed and $g_{s} \equiv g_{s}\left(m_{t} \nu\right)$, $g_{u} \equiv g_{s}\left(m_{t} \nu^{2}\right), g_{s}$ being the QCD coupling. All coefficients are functions of the renormalization parameter $\nu$, and all explicit soft momentum labels are summed over. Massless quarks and ghost terms are not displayed and there are other terms needed for renormalization purposes ${ }^{21,22}$ not shown here. In $d=4-2 \epsilon$ dimensions powers of $\mu_{U}^{\epsilon}$ and $\mu_{S}^{\epsilon}$ are uniquely determined by the mass dimension and the $\mathrm{v}$ counting of each operator. The covariant derivative contains only the ultrasoft gluon field, $D^{\mu}=\partial^{\mu}+i \mu_{U}^{\epsilon} g_{u} A^{\mu}$. The first line in Eq. (3) contains the heavy quark kinetic terms and their interaction with ultrasoft gluons. There are 4-quark potential-like interactions of the form $\left(\mathbf{k}=\left(\mathbf{p}-\mathbf{p}^{\prime}\right)\right)$

$$
V\left(\mathbf{p}, \mathbf{p}^{\prime}\right)=\frac{\mathcal{V}_{c}}{\mathbf{k}^{2}}+\frac{\mathcal{V}_{k} \pi^{2}}{m|\mathbf{k}|}+\frac{\mathcal{V}_{r}\left(\mathbf{p}^{2}+\mathbf{p}^{\prime 2}\right)}{2 m_{t}^{2} \mathbf{k}^{2}}+\frac{\mathcal{V}_{2}}{m_{t}^{2}}+\frac{\mathcal{V}_{s}}{m_{t}^{2}} \mathbf{S}^{2},
$$

where $\mathbf{S}$ is the total $t \bar{t}$ spin operator.

At NNLL order for the total cross section the coefficient $\mathcal{V}_{c}$ of the $1 / \mathbf{k}^{2}$ potential has to be matched at two loops ${ }^{20}$ because it contributes at the LL level, whereas the coefficients of the order $1 / m_{t}^{2}$ potentials only have to matched at the Born level ${ }^{15}$. The $1 /(m|\mathbf{k}|)$-type potentials are of order $\alpha_{s}^{2}$ and have to be matched at two loops. ${ }^{21}$ There are also 4-quark interactions with the radiation of an ultrasoft gluon (last line) and interactions between quarks and soft gluons (second line). Here, due to momentum conservation at least two soft gluons are required. The sum of the potential terms shown in Eq. (4) and time-ordered products of soft interactions contribute to the instantaneous interactions between the top-antitop quark pair (that are traditionally called "potentials").

Particularly important for the phenomenological application are the effective operators involving the top quark width and the residual mass term $\delta m_{t}$ (first line). The width term arises by including the absorptive electroweak contribution from the $\mathrm{W}$-b final state of the top 2-point function into the vNRQCD matching conditions at $\nu=1$. The width is of order $\Gamma_{t} \sim E \sim D^{0} \sim \mathbf{p}^{2} / m_{t} \sim m v^{2}$ and has to be included in the heavy quark propagator. It is this width term which explicitly serves as an IR cutoff and which causes the smooth cross section shape visible in Figs. 1. Techni- 
cally, it corresponds to a shift of the c.m. energy into the positive complex plane by $i \Gamma_{t} \cdot{ }^{23}$ Beyond LL order, however, the structure of electroweak corrections is more complicated. ${ }^{9,24}$ The residual mass term is non-zero in the threshold mass schemes, and cancels the order $\Lambda_{\mathrm{QCD}}$ ambiguity arising from the higher order QCD corrections to the Coulomb potential $\mathcal{V}_{c} / \mathbf{k}^{2}$ that would otherwise destabilize the perturbative behavior of the location where the threshold cross section rises. ${ }^{13}$ (In the pole scheme $\delta m_{t}=0$, and the destabilization due to an $\mathcal{O}\left(\Lambda_{\mathrm{QCD}}\right)$ renormalon takes place. $\left.{ }^{13}\right)$

Besides the interactions contained in the effective Lagrangian that describe the dynamics of the $t \bar{t}$ pair we also need external currents that describe the production of the top quarks. For $e^{+} e^{-}$annihilation these currents are induced by the exchange of a virtual photon or a $\mathrm{Z}$ boson. At NNLL order we need the vector S-wave currents $\mathbf{J}_{\mathbf{p}}^{v}=c_{1}(\nu) \boldsymbol{O}_{\boldsymbol{p}, 1}+$ $c_{2}(\nu) \boldsymbol{O}_{\boldsymbol{p}, 2}$, where $\boldsymbol{O}_{\boldsymbol{p}, 1}=\psi_{\mathbf{p}}^{\dagger} \boldsymbol{\sigma}\left(i \sigma_{2}\right) \chi_{-\mathbf{p}}^{*}, \boldsymbol{O}_{\boldsymbol{p}, 2}=\frac{1}{m^{2}} \psi_{\mathbf{p}}^{\dagger} \mathbf{p}^{2} \boldsymbol{\sigma}\left(i \sigma_{2}\right) \chi_{-\mathbf{p}}^{*}$ and the axial-vector $\mathrm{P}$-wave current $\mathbf{J}_{\mathbf{p}}^{a}=c_{3}(\nu) \boldsymbol{O}_{\boldsymbol{p}, 3}$, where $\boldsymbol{O}_{\boldsymbol{p}, 3}=$ $\frac{-i}{2 m} \psi_{\mathbf{p}}^{\dagger}[\boldsymbol{\sigma}, \boldsymbol{\sigma} \cdot \mathbf{p}]\left(i \sigma_{2}\right) \chi_{-\mathbf{p}}^{*}$. The currents $\boldsymbol{O}_{\boldsymbol{p}, 2}$ and $\boldsymbol{O}_{\boldsymbol{p}, 3}$ lead to contributions in the total cross section that are $v^{2}$-suppressed with respect to those of the current $\boldsymbol{O}_{\boldsymbol{p}, 1}$. Thus, at NNLL order, two-loop matching is needed for $c_{1}$ and Born level matching for $c_{2}$ and $c_{3}$.

\section{The Computations}

The computations for the total cross section in the EFT are performed in the three steps,

i) matching to QCD (including in principle also electroweak interactions) at $\mu_{S}=\mu_{U}=m(\nu=1)$;

ii) determination of the anomalous dimensions and running with the VRG from $\nu=1$ to $\nu=v_{0}$, were $v_{0}$ is the typical heavy quark velocity $\left(v_{0} \approx 0.15\right.$ for $t \bar{t}$ threshold production);

iii) computation of the EFT matrix elements at $\nu=v_{0}$.

Conceptually, the novel aspect of the computations is the summation of the logarithms of the correlated soft and ultrasoft scales using the VRG. Although these calculations are usual standard once renormalization is expressed in terms of the renormalization parameter $\nu$, they are non-trivial conceptually. This is because the correlation of soft and ultrasoft renormalization scales in the evolution is crucial to achieve the proper summation of logarithms. This was demonstrated in QED, ${ }^{25}$ where higher order logarithmic terms could only be reproduced correctly using the VRG. A 
nice demonstration at the technical level on the difference between a correlated and an uncorrelated running was given $i^{26}$. Technically the necessity of correlated running arises whenever divergences in massive quark loops and ultrasoft or soft loops contribute simultaneously to an anomalous dimension. It was also shown ${ }^{17,22}$ that subdivergences in the NNLL (3-loop) renormalization of the current $\boldsymbol{O}_{\boldsymbol{p}, 1}$, arising from diagrams where UV-divergences from massive quark and ultrasoft or soft loops arise simultaneously in one single diagram, could be treated consistently in the VRG, while an uncorrelated treatment of soft and ultrasoft renormalization fails. Through steps i) and ii) all logarithmic terms involving $v$ are summed into the operator coefficients and the matrix elements in iii) involve $\ln (v / \nu)$ terms that are not parametrically large.

As far as QCD effects are concerned, for the renormalization group improved $t \bar{t}$ threshold cross section in vNRQCD all necessary components in the steps i), ii) and iii) are known at NNLL order with the exception of step ii) for the current coefficient $c_{1}$. The running of the potential coefficients $\mathcal{V}_{i}$ was determined in Refs. ${ }^{15,20,27,21}$ and of the current coefficients $c_{i}$ in Refs. ${ }^{27,21}$. Up to notational differences there is agreement with the potential coefficients obtained in pNRQCD. ${ }^{28}$ The running of $c_{1}$ is fully known at NLL order, ${ }^{27,21}$ while at NNLL order there is still some work to do. The full set of non-mixing contributions coming from UV-divergences in actual three-loop vertex diagrams have been determined $i^{22}$. A nontrivial cross check for this computation, which checked the $\alpha_{s}^{3} \ln \nu$ term in $c_{1}(\nu)$ was also carried out. ${ }^{29}$ The full set of mixing contributions, which arise from the next-order running of the Wilson coefficients appearing in the NLL anomalous dimension of $c_{1}$ is still unknown. Recently, the mixing contribution arising from the spin-dependent $\mathcal{O}\left(1 / m_{t}^{2}\right)$ potential (last term in Eq. (4) was computed in NRQCD-pNRQCD. ${ }^{30}$

\section{The Top Pair Total Cross Section at Threshold}

The total cross section for $e^{+} e^{-} \rightarrow \gamma^{*}, Z^{*} \rightarrow t \bar{t}$ at threshold at NNLL order in renormalization group improved perturbation theory has the form

$$
\sigma_{\text {tot }}^{\gamma, Z}(s)=\frac{4 \pi \alpha^{2}}{3 s}\left[F^{v}(s) R^{v}(s)+F^{a}(s) R^{a}(s)\right],
$$

where $F^{v, a}$ are trivial functions of the electric charges and the isospin of the electron and the top quark and of the weak mixing angle. The vector 
and axial-vector $R$-ratios have the form ${ }^{17}$

$$
\begin{aligned}
& R^{v}(s)=\frac{4 \pi}{s} \operatorname{Im}\left[c_{1}^{2}(\nu) \mathcal{A}_{1}(v, m, \nu)+2 c_{1}(\nu) c_{2}(\nu) \mathcal{A}_{2}\left(v, m_{t}, \nu\right)\right], \\
& R^{a}(s)=\frac{4 \pi}{s} \operatorname{Im}\left[c_{3}^{2}(\nu) \mathcal{A}_{3}\left(v, m_{t}, \nu\right)\right]
\end{aligned}
$$

where the $\mathcal{A}_{i}$ 's are T-products of the effective theory currents described before. As mentioned before, the running of $c_{1}$ is only partly know at NNLL order. These T-products are related to the zero-distance Green function
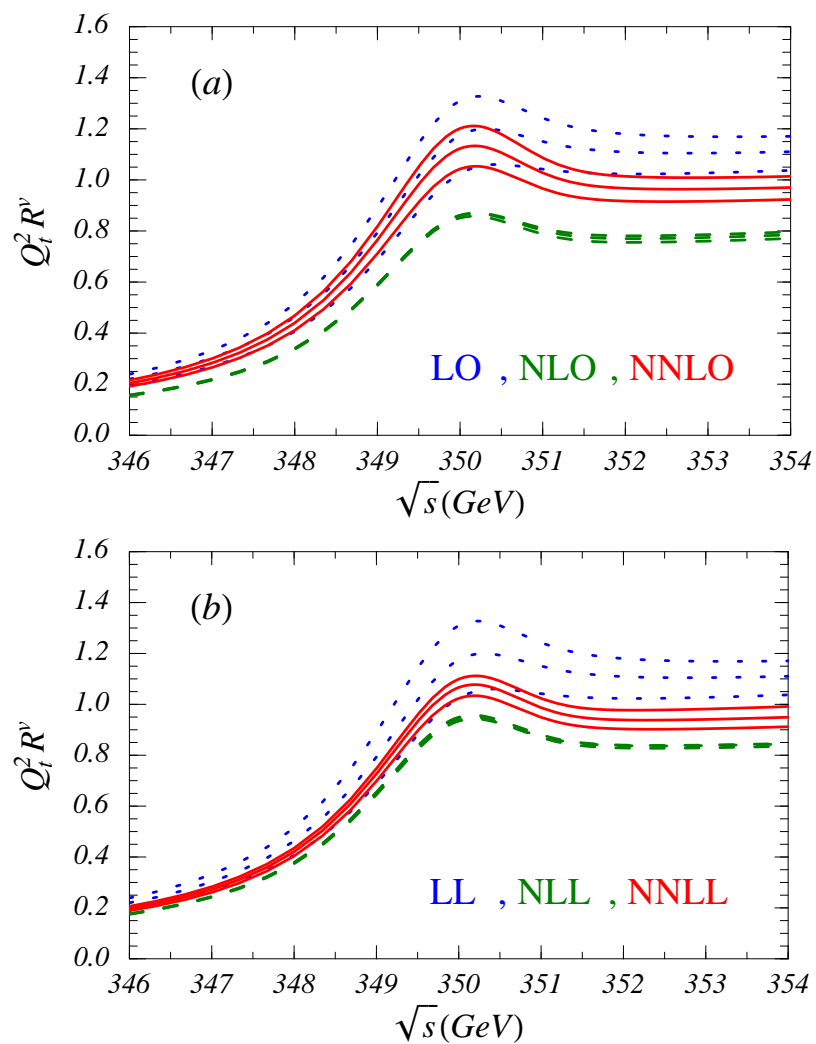

Figure 1. Panel a) shows the results for $Q_{t}^{2} R^{v}$ with $M^{1 \mathrm{~S}}=175 \mathrm{GeV}$ and $\Gamma_{t}=1.43 \mathrm{GeV}$ in fixed-order perturbation theory at LO (dotted lines), NLO (dashed lines) and NNLO (solid lines). Panel b) shows the results for $Q_{t}^{2} R^{v}$ with the same parameters in renormalization group improved perturbation theory at LL (dotted lines), NLL (dashed lines) and NNLL (solid lines) order. For each order curves are plotted for $\nu=0.15,0.20$, and 0.3 . The effects of initial state radiation, beamstrahlung and the beam energy spread at a $e^{+} e^{-}$collider are not included. The plots are from Ref.[32]. 
of the equation of motion of the 4-quark 4-point functions obtained in the EFT. (At NNLL order this is a common 2-body Schrödinger equation, since higher Fock quark-antiquark-gluon states only contribute to the renormalization of operators.) I use the $1 \mathrm{~S}$ mass ${ }^{9,31}$ since it is the threshold mass that is most closely related to the peak position visible in the theoretical prediction. Other threshold masses are also viable ${ }^{13}$ but not discussed here further.

In Fig. 1b I have displayed the photon induced cross section $Q_{t}^{2} R^{v}$ at LL (dotted blue lines), NLL (dashed green lines) and NNLL (solid red lines) order for $\nu=0.15,0.2$ and 0.3. Figure 1a shows the corresponding results in fixed-order perturbation theory already discussed earlier. Compared to the fixed-order results with the same scales the improvement is substantial, particularly around the peak position and for smaller energies, but the somewhat inconvenient behavior that the NLL and the NNLL order predictions do not overlap remains. At present the uncertainty of the normalization of the total cross section is $\delta \sigma_{t \bar{t}} / \sigma_{t \bar{t}} \simeq \pm 6 \%$ due to this visibly large shift between the NLL and the NNLL order predictions. ${ }^{32}$ In fact, an uncertainty not larger than $3 \%$ would be required to have the theoretical errors of measurements of the top Yukawa coupling, $\Gamma_{t}$ and $\alpha_{s}$ match with the ones presently expected from experiments. ${ }^{2}$ It is, however, premature to draw definite conclusions as long as the NNLL mixing contributions to $c_{1}$ have not yet been determined completely.

\section{Acknowledgement}

I would like to thank the organizers of the conference for the hospitality and the pleasant atmosphere at the Theoretical Physics Institute.

\section{References}

1. R. D. Heuer, D. J. Miller, F. Richard and P. M. Zerwas, hep-ph/0106315. T. Abe et al. [American Linear Collider Working Group Collaboration], in Proc. of the APS/DPF/DPB Summer Study on the Future of Particle Physics (Snowmass 2001), ed. N. Graf, arXiv:hep-ex/0106057.

2. M. Martinez and R. Miquel, Eur. Phys. J. C 27, 49 (2003) [arXiv:hep$\mathrm{ph} / 0207315]$.

3. W.E. Caswell and G.P. Lepage, Phys. Lett. 167B, 437 (1986). G.T. Bodwin, E. Braaten and G.P. Lepage, Phys. Rev. D51, 1125 (1995), ibid. D55, 5853 (1997).

4. A. H. Hoang and T. Teubner, Phys. Rev. D58, 114023 (1998) [hep$\mathrm{ph} / 9801397]$. 
5. K. Melnikov and A. Yelkhovsky, Nucl. Phys. B528, 59 (1998) [hep$\mathrm{ph} / 9802379]$

6. O. Yakovlev, Phys. Lett. B457, 170 (1999) [hep-ph/9808463].

7. M. Beneke, A. Signer and V. A. Smirnov, Phys. Lett. B454, 137 (1999) [hep-ph/9903260].

8. T. Nagano, A. Ota and Y. Sumino, Phys. Rev. D60, 114014 (1999) [hepph/9903498].

9. A. H. Hoang and T. Teubner, Phys. Rev. D60, 114027 (1999) [hep$\mathrm{ph} / 9904468]$.

10. A. A. Penin and A. A. Pivovarov, Nucl. Phys. B550, 375 (1999) [hepph/9810496]; Phys. Atom. Nucl. 64, 275 (2001) [Yad. Fiz. 64, 323 (2001)] [hep-ph/9904278].

11. M. Battaglia et al., arXiv:hep-ph/0304132.

12. D. Peralta, M. Martinez and R. Miquel, talk presented at the 4th International Workshop on Linear Colliders, Sitges, Barcelona, Spain, April 28 May 51999.

13. A. H. Hoang et al., in Eur. Phys. J. direct C3, 1 (2000) [hep-ph/0001286].

14. M. Luke, A. Manohar and I. Rothstein, Phys. Rev. D61, 074025 (2000) [arXiv:hep-ph/9910209].

15. A.V. Manohar and I.W. Stewart, Phys. Rev. D 62, 014033 (2000) [arXiv:hep$\mathrm{ph} / 9912226]$.

16. A.V. Manohar and I.W. Stewart, Phys. Rev. D62, 074015 (2000) [arXiv:hep$\mathrm{ph} / 0003032]$.

17. A. H. Hoang, A. V. Manohar, I. W. Stewart and T. Teubner, Phys. Rev. D 65, 014014 (2002) [arXiv:hep-ph/0107144].

18. N. Brambilla, A. Pineda, J. Soto and A. Vairo, Nucl. Phys. B 566, 275 (2000) [arXiv:hep-ph/9907240].

19. A. Pineda, Phys. Rev. D 66, 054022 (2002) [arXiv:hep-ph/0110216].

20. A.H. Hoang, A.V. Manohar and I.W. Stewart, Phys. Rev. D 64, 014033 (2001) [arXiv:hep-ph/0102257].

21. A. H. Hoang and I. W. Stewart, Phys. Rev. D 67, 114020 (2003) [arXiv:hep$\mathrm{ph} / 0209340]$.

22. A. H. Hoang, Phys. Rev. D 69, 034009 (2004) [arXiv:hep-ph/0307376].

23. V. S. Fadin and V. A. Khoze, JETP Lett. 46, 525 (1987).

24. A. H. Hoang and C Reißer, in preparation.

25. A. V. Manohar and I. W. Stewart, Phys. Rev. Lett. 85, 2248 (2000) arXiv:hep-ph/00004018;

26. A. V. Manohar, J. Soto and I. W. Stewart, Phys. Lett. B 486, 400 (2000) arXiv:hep-ph/0006096;

27. A.V. Manohar and I.W. Stewart, Phys. Rev. D63, 054004 (2001). [arXiv:hepph/0003107].

28. A. Pineda, Phys. Rev. D 65, 074007 (2002) [arXiv:hep-ph/0109117].

29. B. A. Kniehl, A. A. Penin, M. Steinhauser and V. A. Smirnov, Phys. Rev. Lett. 90, 212001 (2003) [arXiv:hep-ph/0210161].

30. A. A. Penin, A. Pineda, V. A. Smirnov and M. Steinhauser, Nucl. Phys. B 699, 183 (2004) [arXiv:hep-ph/0406175]. 
31. A. H. Hoang, Z. Ligeti and A. V. Manohar, Phys. Rev. Lett. 82, 277 (1999) [hep-ph/9809423]; Phys. Rev. D59, 074017 (1999) [hep-ph/9811239].

32. A. H. Hoang, Acta Phys. Polon. 34, 4491 (2003) [arXiv:hep-ph/0310301]. 